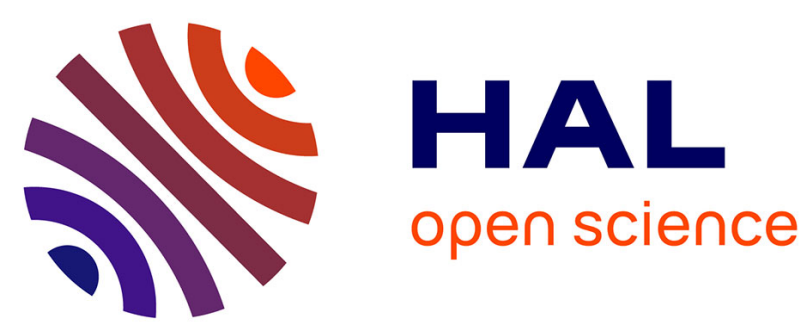

\title{
PROPRIÉTÉS MAGNÉTIQUES DE L'OXYDE DE COBALT CoO EN GRAINS FINS
}

\author{
P. Mollard, F. de Bergevin, P. Germi, F. Vincent, M. Figlarz
}

\section{To cite this version:}

P. Mollard, F. de Bergevin, P. Germi, F. Vincent, M. Figlarz. PROPRIÉTÉS MAGNÉTIQUES DE L'OXYDE DE COBALT CoO EN GRAINS FINS. Journal de Physique Colloques, 1971, 32 (C1), pp.C1-1041-C1-1042. 10.1051/jphyscol:19711373 . jpa-00214411

\section{HAL Id: jpa-00214411 https://hal.science/jpa-00214411}

Submitted on 1 Jan 1971

HAL is a multi-disciplinary open access archive for the deposit and dissemination of scientific research documents, whether they are published or not. The documents may come from teaching and research institutions in France or abroad, or from public or private research centers.
L'archive ouverte pluridisciplinaire HAL, est destinée au dépôt et à la diffusion de documents scientifiques de niveau recherche, publiés ou non, émanant des établissements d'enseignement et de recherche français ou étrangers, des laboratoires publics ou privés. 


\title{
PROPRIÉTÉS MAGNÉTIQUES DE L'OXYDE DE COBALT CoO EN GRAINS FINS
}

\author{
P. MOLLARD, F. de BERGEVIN, P. GERMI \\ Laboratoire d'Electrostatique et de Physique du Métal \\ C. N. R. S., CEDEX 166, Grenoble, France \\ F. VINCENT, M. FIGLARZ \\ Laboratoire de Chimie des Solides, Sorbonne, Paris, France
}

Résumé. - Les auteurs indiquent, à partir d'une méthode permettant d'obtenir la distribution des températures de blocage d'une assemblée de grains fins antiferromagnétiques, la variation de la température de blocage moyenne avec la dimension moyenne des cristallites de $\mathrm{CoO}$. Le produit a été obtenu par déshydratation topotactique de $\mathrm{Co}(\mathrm{OH})_{2}$. Les propriétés physico-chimiques ont été analysées à partir du profil des raies de diffraction aux rayons $\mathrm{X}$, par microscopie et diffraction électronique, thermogravimétrie et spectrographie infrarouge.

Abstract. - Using a method giving the distribution of the blocking temperatures of an assembly of antiferromagnetic fine particles we have determinated the variation of the average blocking temperature as a function of the average dimension of the $\mathrm{CoO}$ crystallites. The oxide has been obtained from topotactic dehydration of $\mathrm{Co}(\mathrm{OH})_{2}$. Its structure has been analysed from the profile of X-Rays diffraction lines, electron microscopy, electron diffraction, thermogravimetry and infrared spectroscopy.

I. - L'interprétation des propriétés magnétiques des antiferromagnétiques finement divisés a été donnée par L. Néel [1] et une revue bibliographique faite par Jacobs et Beans [2]. La théorie met en relief la présence d'une température de blocage variable avec la dimension des cristallites au-dessous d'un diamètre critique et la présence d'une susceptibilité magnétique additionnelle dans le domaine d'ordre due à des effets de surface: le superantiferromagnétisme [3]. Nous avons indiqué antérieurement une méthode permettant d'obtenir à l'aide de mesures magnétostatiques la distribution des températures de blocage d'une assemblée de grains fins antiferromagnétiques [4]. Nous indiquons ici les résultats les plus caractéristiques obtenus sur CoO.

II. - La déshydratation sous argon purifié de $\mathrm{Co}(\mathrm{OH})_{2}$ conduit à des grains élémentaires de $\mathrm{CoO}$ qui sont des plaquettes hexagonales poreuses monocristallines mosaïques [5] de dimensions moyennes $2000 \times 2000 \times 300 \AA$ et de plan de base (111) et dont la taille des cristallites est fonction de la température de préparation.

La spectrographie infrarouge [6] montre la présence d'hydroxyles libres et liés superficiels et d'eau moléculaire.

Le tableau I donne les dimensions moyennes des cristallites, obtenues soit â partir de l'élargissement à

\section{TABleau I}

\begin{tabular}{|c|c|c|c|}
\hline Poudre & $d_{\mathrm{f}}$ & $d_{\mathrm{m}}$ & $<T_{\mathrm{B}}>{ }^{\circ} \mathrm{K}$ \\
\hline $\mathrm{G}_{1}$ & $300 \AA$ & $300 \AA$ & $\sim T_{\mathrm{N}}$ \\
\hline $\mathrm{G}_{2}$ & $90 \AA$ & $110 \AA$ & 289,5 \\
\hline $\mathrm{G}_{4}$ & $35 \AA$ & $60 \AA$ & 240 \\
\hline $\mathrm{G}_{7}$ & $45 \AA$ & $60 \AA$ & 232 \\
\hline $\mathrm{G}_{8}$ & $75 \AA$ & 85-90 $\AA$ & 265 \\
\hline $\mathrm{G}_{9}$ & $35 \AA$ & $50 \AA$ & 218 \\
\hline $\mathrm{G}_{10}$ & $40 \AA$ & $60 \AA$ & 227 \\
\hline $\mathrm{G}_{11}$ & $50 \AA$ & $70 \AA$ & 242 \\
\hline
\end{tabular}

mi-hauteur des raies de diffraction (dm) soit à partir de l'analyse des profils de raies (df). La figure 1 donne la répartition granulométrique [7] obtenue sur l'échantillon $\mathrm{G}_{4}$. Cette double distribution est caractéristique des échantillons étudiés. La distribution inférieure correspond à une perturbation du fond continu idu diagramme $\mathrm{X}$ due à la présence de lacunes figure 1 [8]. La valeur moyenne de la deuxième distribution est pratiquement $d_{m}$.

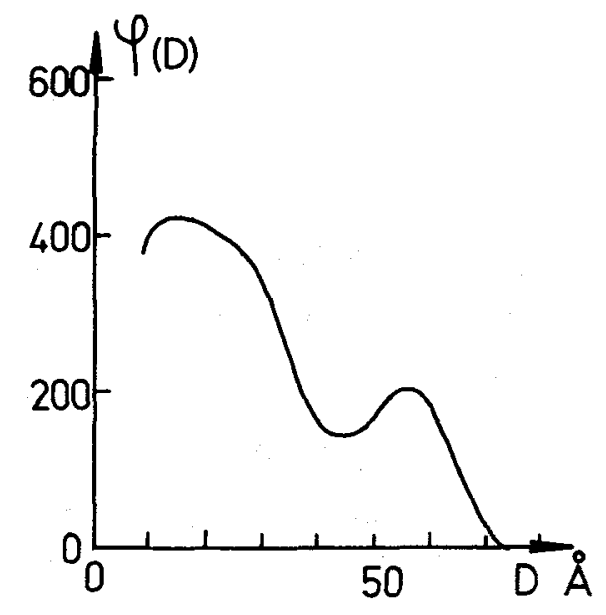

FIG. 1. - Fonction de répartition du diamètre des cristallites (raie 200 ), $\mathrm{G}_{4}$.

III. - Nous avons déduit de la variation thermique du moment magnétique après refroidissement en champ magnétique nul et après refroidissement depuis $T_{\mathrm{a}}$ jusqu'à $T_{0}$ dans un champ $H_{\mathrm{r}}$ l'évolution de l'aimantation thermorémanente (A. T. R.) partielle $\sigma_{T}\left(T_{0}, H_{\mathrm{r}}\right.$, $T_{\mathrm{a}}$ ) acquise dans $H_{\mathrm{r}}$ par les cristallites dont $T_{\mathrm{B}}$ est dans l'intervalle $\left(T_{0}, T_{\mathrm{a}}\right)$, figure 2. $\sigma_{T_{0}}\left(T_{0}, H_{\mathrm{r}}, T_{\mathrm{a}}\right)$ permet d'obtenir l'A. T. R. des cristallites dont $T_{\mathrm{B}}$ est dans l'intervalle $\Delta T_{\mathrm{B}}$ et donne ainsi une image de la distribu- 


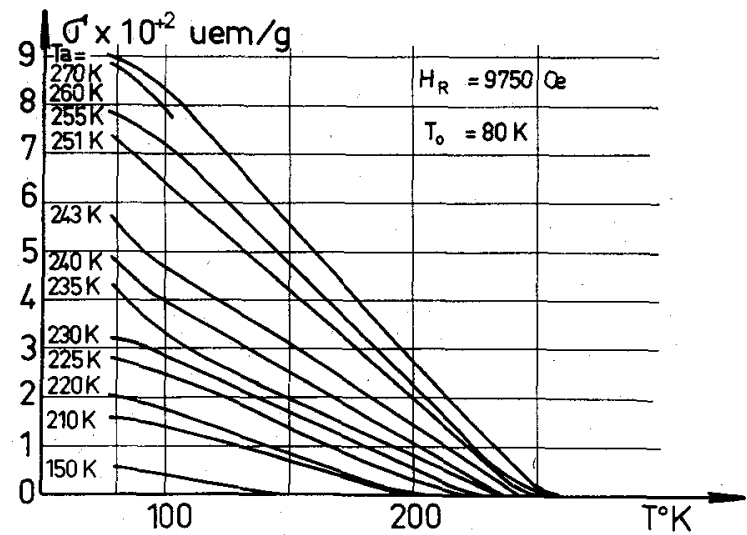

FIG. 2. - Variation thermique des aimantations thermorémanentes partielles $\sigma 2\left(80,9750, T_{\mathrm{a}}\right), \mathrm{G}_{4}$.

tion des températures de blocage (Fig. 3) et la température de blocage moyenne.

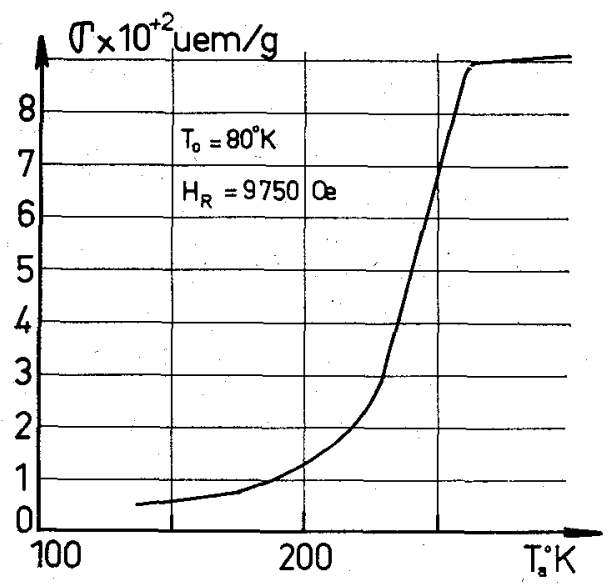

Fig. 3. - Variation de $1^{\prime} \mathrm{A}$. T. R. à $80^{\circ} \mathrm{K}$ en fonction de la température $T_{\mathrm{a}}$ de refroidissement sous champ magnétique $\sigma_{80}\left(80,9750, T_{2}\right), \mathrm{G}_{4}$.

IV. - La faiblesse de l'augmentation de la susceptibilité superantiferromagnétique ( 20 à $30 \%$ ), figure 4 , confirme la théorie de L. Néel [3] tenant compte des couplages magnétocristallins; l'énergie d'anisotropie étant très forte dans $\mathrm{CoO}$ seuls les plans réticulaires

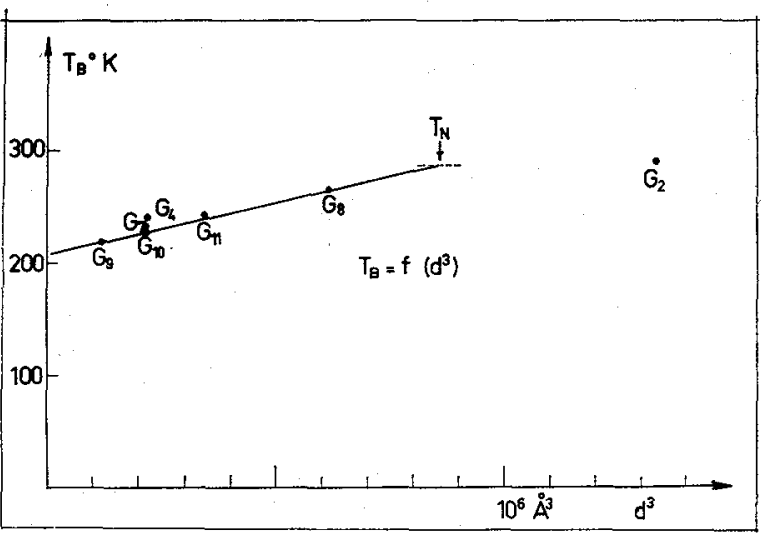

FIg. 4. - Variation thermique de la susceptibilité magnétique après refroidissement en champ nul et après refroidissement sous champ magnétique, $\mathbf{G}_{4}$.

actifs superficiels interviennent. Les températures de blocage élevé observées confirment la valeur élevée de l'énergie d'anisotropie. La courbe de répartition granulométrique de $\mathrm{G}_{2}$ permet de chiffrer à $80 \AA$ la dimension de $d$ pour laquelle $T_{\mathrm{B}} \# T_{\mathrm{N}}$. La température de blocage moyenne varie comme $d_{\mathrm{m}}^{3}$ (Fig. 5), ce qui est en faveur d'une énergie de blocage en volume : une confirmation indirecte de ceci réside dans le fait que les distributions observées des températures de blocage ne s'étalent pas entre $0^{\circ} \mathrm{K}$ et $\left\langle T_{\mathrm{B}}\right\rangle$ comme dans le cas d'une énergie de blocage d'origine superficielle $[1,9]$.

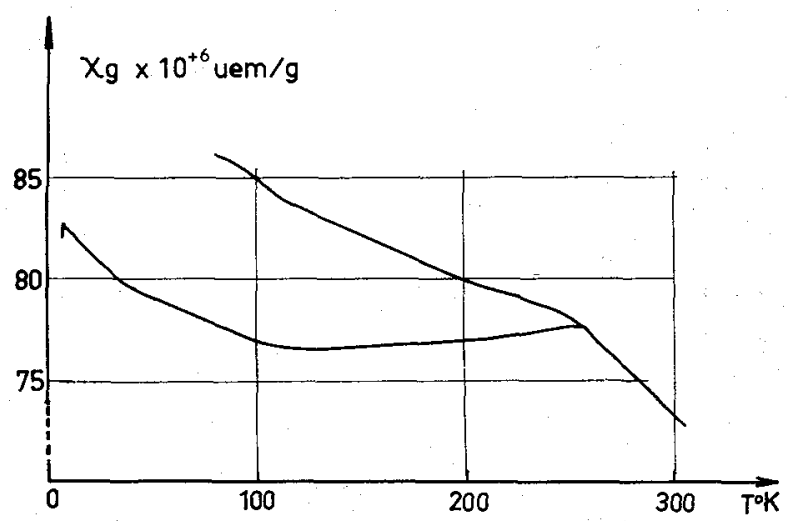

Fig. 5. - Variation thermique de $\left\langle T_{\mathrm{B}}\right\rangle$ avec $d_{\mathbf{m}}^{3}$.

\section{Bibliographie}

[1] Néel (L.), Cours, les Houches, 1961, P. U. F. Paris. [2] Jacobs (I. S.), BeAns (C. P.), Magnetism, 1963, 3, Ac. Press. Inc. New York.

[3] Néel (L.), C. R. Acad. Sci., Paris, 1962, 254, 598.

[4] Mollard (P. ), Figlarz (M.), Vincent (F.), $C . R$. Acad. Sci., Paris, 1969, 269B, 448.

[5] Figlarz (M.), Vincent (F.), C. R. Acad. Sci., Paris, $1968,266 \mathrm{C}, 376$.
[6] Vincent (F.), Figlarz (M.), Amiel (J.), Proc. 6th Int. Symp. on the Reactivity of Solids, New York, J. Wiley, 1969, 181.

[7] Germi (P.), de BerGevin (F.), A paraître.

[8] Willis (B. T. M.), Chem, of Ext defects in nonmetallic solids, North Holland Publishing Co, $1970,272$.

[9] Etude faite en partie dans le cadre du contrat D.R.M. E., $n^{0} 321-68$. 